\title{
Prophylactic colectomy for children with familial adenomatous polyposis: resource utilization and outcomes for open and laparoscopic surgery
}

\author{
Anthony Ferrantella ${ }^{1}$, Rebecca A. Saberi ${ }^{1}$, Brent A. Willobee ${ }^{1}$, Hallie J. Quiroz ${ }^{1}$, Amber H. Langshaw ${ }^{2}$, \\ Samir Pandya ${ }^{3}$, Chad M. Thorson ${ }^{1}$, Juan E. Sola ${ }^{1}$, Eduardo A. Perez ${ }^{1}$ \\ ${ }^{1}$ Division of Pediatric Surgery, Department of Surgery, ${ }^{2}$ Division of Pediatric Gastroenterology, Department of Pediatrics, University of Miami \\ Miller School of Medicine, Miami, FL, USA; ${ }^{3}$ Division of Pediatric Surgery, Department of Surgery, UT Southwestern Medical Center, Dallas, \\ TX, USA \\ Contributions: (I) Conception and design: All authors; (II) Administrative support: All authors; (III) Provision of study materials or patients: All \\ authors; (IV) Collection and assembly of data: All authors; (V) Data analysis and interpretation: All authors; (VI) Manuscript writing: All authors; (VII) \\ Final approval of manuscript: All authors. \\ Correspondence to: Eduardo A. Perez, MD. Associate Professor, Division of Pediatric Surgery, DeWitt Daughtry Family Department of Surgery, \\ University of Miami Leonard M. Miller School of Medicine, 1120 NW 14th Street, Suite 450K, Miami, FL 33136, USA. \\ Email: EPerez3@med.miami.edu.
}

Background: Laparoscopic approach for the surgical management of familial adenomatous polyposis (FAP) has become increasingly common for pediatric patients. The purpose of this study was to compare shortterm outcomes and resource utilization between open and laparoscopic surgery for prophylactic colectomy in children with FAP.

Methods: The Kids' Inpatient Database (2009 and 2012) was analyzed for children (age $\leq 20$ years) with FAP that underwent prophylactic total colectomy or proctocolectomy. Patient demographics, treating hospital characteristics, hospital charges, and short-term outcomes were compared according to the surgical technique utilized (open versus laparoscopic).

Results: Overall, we identified 216 patients with FAP that underwent elective total colectomy, of which 95 cases were performed by open surgery and 121 were done laparoscopically. The majority of patients were treated at large, not-for-profit, urban teaching hospitals, and the median age was equal (16 years) in both groups. Complications that were more common for open procedures included accidental perforation or hemorrhage ( $4 \%$ vs. $0 \%, \mathrm{P}=0.023)$, reopening of surgical site ( $3 \%$ vs. $0 \%, \mathrm{P}=0.049)$, and pneumonia ( $3 \%$ vs. $0 \%, \mathrm{P}=0.049)$. Simultaneous proctectomy was performed more commonly in the open cohort $(91 \%$ vs. $71 \%$, $\mathrm{P}<0.001)$ as well as ileostomy creation $(74 \%$ vs. 49\%, $\mathrm{P}<0.001)$. The median length of stay was similar in the open and laparoscopic groups (7 vs. 6 days, $\mathrm{P}=0.712)$. Median total hospital charges were also similar $(\$ 67,334$ vs. $\$ 68,717, \mathrm{P}=0.080)$.

Conclusions: A laparoscopic approach for prophylactic colectomy can be safely performed in children with FAP, and total hospital charges are equivalent compared to open surgery. However, simultaneous proctectomy was performed less often with laparoscopic surgery.

Keywords: Pediatric; laparoscopic surgery; resource utilization; familial adenomatous polyposis (FAP)

Received: 16 April 2020; Accepted: 15 July 2020; Published: 25 July 2021.

doi: $10.21037 /$ tgh-20-190

View this article at: http://dx.doi.org/10.21037/tgh-20-190 


\section{Introduction}

Familial adenomatous polyposis (FAP) results in the formation of thousands of adenomatous polyps in the colon and rectum early in life, and if left untreated, inevitable progression to colorectal cancer by age $40-50$ years due to nearly $100 \%$ disease penetrance (1-3). Given the high risk of colorectal malignancy associated with FAP, management protocols suggest that screening should begin around ages $10-14$ years (1-3). Once polyposis is identified, prophylactic colectomy or proctocolectomy is generally recommended, with the timing and type of surgery dependent upon disease severity, patient age, and psychosocial factors (2).

The most common surgical procedures for the management of FAP include total abdominal colectomy with ileorectal anastomosis, restorative proctocolectomy with ileal pouch-anal anastomosis, and total proctocolectomy with end ileostomy (4-6). Restorative proctocolectomy with ileal pouch-anal anastomosis has become the mainstay of surgical management, and a laparoscopic approach has gained significant support in recent years $(2,4,5,7,8)$. Jacobs et al. initially described laparoscopic colectomy in 1991, while the first prophylactic laparoscopic ileorectal anastomosis for FAP was reported in 1997 by Milsom et al. (6). Many studies have since compared laparoscopic versus conventional open ileal pouch-anal anastomosis and ileorectal anastomosis to determine the feasibility of laparoscopy in the setting of prophylactic surgery for the management of FAP (2-7,9-14). In comparison to an open approach, benefits of laparoscopic surgery may include shorter length of stay $(3,4,10,11,13,14)$, less need for intra-operative blood transfusion $(5,6,8,14)$, lower rate of desmoid tumors post-operatively $(3,9)$ decreased incidence of post-operative superficial surgical site infection $(3,7,10)$, and a lower rate of minor, short-term complications $(4,7,11,13)$ with no increase in major, longterm complications (11). However, many of these analyses included adult patients, cohorts consisting of both patients with FAP and ulcerative colitis, and/or were small singleinstitution studies and are thus not widely generalizable to the pediatric patient population.

While evidence supports that a laparoscopic approach for elective, prophylactic treatment of FAP in pediatric patients is at least as safe as open technique, the data regarding possible advantages of a minimally invasive approach remain limited $(2,3,4-6,8,11,13,14)$. Alternatively, operative duration has consistently been found to be longer with a laparoscopic approach, which is reportedly associated with higher overall costs $(4,7,8,13,14)$. Further investigation is needed to determine if the outcomes following laparoscopic surgery provide an advantage significant enough to justify the potential increased costs of the procedure for this patient population. Therefore, this study aims to evaluate the clinical outcomes and resource utilization associated with open versus laparoscopic surgery for prophylactic total abdominal colectomy and proctocolectomy in pediatrics patients with FAP. We hypothesized that laparoscopic surgery is associated with improved short-term outcomes and similar hospital resource utilization. We present the following article in accordance with the STROBE reporting checklist (available at http://dx.doi.org/10.21037/tgh-20-190).

\section{Methods}

Nationwide data on U.S. children with FAP that underwent colectomy in the years 2009 and 2012 were obtained from the Agency for Healthcare Research and Quality (AHRQ)sponsored Healthcare Cost and Utilization Project (HCUP) Kids' Inpatient Database (KID). The KID is the largest publicly available all-payer pediatric inpatient care database in the U.S. and is released on a three-year cycle. It contains data from approximately 3 million pediatric discharges each year across 44 different states and is weighted for national estimates of over 7 million hospitalizations. Therefore, the KID is a powerful tool to analyze national trends in healthcare utilization, access, charges, quality, and outcomes for both common and rare pediatric conditions (15).

Children (age $\leq 20$ years) with a diagnosis of FAP were identified in the KID by the International Classification of Disease, Ninth Revision (ICD-9) diagnosis codes 211.3 and 211.4. Patients who underwent an operation for total abdominal colectomy were identified by the Current Procedural Terminology (CPT) codes 45.81 and 45.82, which differentiate between procedures performed by an open or laparoscopic approach. Cases containing both of these CPT codes were excluded. Simultaneous proctectomy and/or creation of an ileostomy were determined using the following secondary CPT codes: 45.92, 45.93, 45.95, 46.20, $46.21,46.23$, 48.40, 48.42, 48.43, 48.49, 48.50, 48.51, $48.52,48.63,48.69$. In order to identify those children undergoing prophylactic surgery, we excluded patients that underwent surgery during non-elective admissions as well as patients with a concurrent diagnosis code for colorectal malignancy.

The patient demographics, concurrent diagnoses, length of stay, treating hospital characteristics, and total hospital charges were compared. Categorical variables were 
compared using a chi-squared test, and continuous variables with nonparametric distributions were compared using the Mann-Whitney test. Significance was set at $\mathrm{P}<0.05$. Results were weighted for national estimates according to HCUP standards based upon treating hospital characteristics and patient factors (15). The study was conducted in accordance with the Declaration of Helsinki (as revised in 2013). The Institutional Review Board of the University of Miami waived the requirement for approval of this study since the KID contains de-identified, publicly available data and is not considered human subjects research. Statistical analyses were performed using SPSS Statistics version 24, IBM Corporation, Armonk, New York.

\section{Results}

Overall, we identified 256 patients with FAP that underwent total abdominal colectomy. We excluded 33 patients that underwent surgery during non-elective admissions and 7 patients that carried a diagnosis of malignancy. Of the remaining 216 patients, 95 underwent open colectomy and 121 underwent laparoscopic colectomy (Table 1). The median age was similar (16 years) for both groups, and sex did not differ significantly between the two groups. Additionally, there were no differences in the household income levels or primary payer status for these patients. In both groups, the majority of patients were treated at large, not-for-profit, urban teaching hospitals.

Wound infection rates were similar between both groups (6\% open vs. $5 \%$ lap, $\mathrm{P}=0.666$, Table 2), but patients in the open colectomy cohort were more likely to require reopening of the surgical site $(3 \%$ open vs. $0 \%$ lap, $\mathrm{P}=0.049$ ). Pneumonia was also more common in the open group ( $3 \%$ open $v$ s. $0 \%$ lap, $\mathrm{P}=0.049)$. However, there was no significant difference in the rates of sepsis or bacteremia ( $2 \%$ open $v$ s. $1 \%$ lap, $\mathrm{P}=0.437$ ). The frequency of electrolyte and fluid disturbances was comparable for both groups ( $9 \%$ open vs. $6 \%$ lap, $\mathrm{P}=0.304$ ), as was the occurrence of postoperative gastrointestinal disorders (3\% open $v s .5 \%$ lap, $\mathrm{P}=0.511)$. Only one patient from the laparoscopic cohort acquired an acute deep venous thrombosis $(0 \%$ open vs. $1 \%$ lap, $\mathrm{P}=0.374)$. Despite more patients in the open surgery group being identified with coagulopathy (3\% open vs. $0 \%$ lap, $\mathrm{P}=0.049$ ), the frequency of patients requiring blood transfusions ( $5 \%$ open vs. $7 \%$ lap, $\mathrm{P}=0.679$ ) or experiencing gastrointestinal bleeding ( $2 \%$ open $v$ s. $1 \%$ lap, $\mathrm{P}=0.431$ ) did not differ significantly between the open and laparoscopic cohorts. Patients undergoing open colectomy were more likely to have an accidental perforation or hemorrhage (4\% open vs. $0 \%$ lap, $\mathrm{P}=0.023$ ) coded during their admission. There were no in-hospital deaths for either group.

In the open colectomy cohort, patients were more likely to undergo simultaneous proctectomy (91\% open vs. $71 \%$ lap, $\mathrm{P}<0.001$, Table 3) as well as ileostomy creation $(74 \%$ open vs. $49 \%$ lap, $\mathrm{P}<0.001)$. The median length of stay was similar in the open and laparoscopic groups (7 days open $v s$. 6 days lap, $\mathrm{P}=0.712$ ). Sub-group analyses comparing length of stay for subsets of surgical procedures demonstrated no significant difference between open and laparoscopic surgery regardless of whether or not ileostomy creation was performed (data not shown). Similarly, length of stay was equivalent for open and laparoscopic proctocolectomy. However, median length of stay for laparoscopic total colectomy (without proctectomy) was reduced compared to open total colectomy ( 9 days open $v$ s. 6 days lap, $\mathrm{P}=0.002$ ). There was no significant difference in the median total hospital charges ( $\$ 67,334$ open vs. $\$ 68,717$ lap, $\mathrm{P}=0.080$ ).

\section{Discussion}

We employed this large administrative data set to characterize the surgical management surrounding a rare condition among children. Whereas prior studies have been limited by single-institution experiences and substantial heterogeneity with regard to the patients included, the current study focuses on the outcomes of pediatric patients undergoing prophylactic surgery to mitigate the risks of colorectal malignancy associated with FAP. By excluding patients that underwent procedures during non-elective admissions as well as those diagnosed with colorectal malignancy, our methodology ensures that the study population includes only those children undergoing planned, prophylactic surgical resections in an effort to truly compare open and laparoscopic techniques for this specific indication.

In our current study, the overall rates of postoperative complications are quite low for both open and laparoscopic approaches in comparison to prior studies evaluating the outcomes between open and laparoscopic total colectomy and proctocolectomy. This difference may be, at least in part, due to the exclusion of patients with ulcerative colitis or Crohn's disease as many of those patients require immunosuppressive medical treatments, which have been shown to correlate with increased rates of complications in patients undergoing these procedures $(11,16,17)$. 
Table 1 Demographics and clinical features of patients undergoing prophylactic colectomy for FAP

\begin{tabular}{|c|c|c|c|}
\hline Features & Open $(\mathrm{N}=95)$ & Lap $(\mathrm{N}=121)$ & $P$ value \\
\hline Sex & & & 0.193 \\
\hline Female & $54(57 \%)$ & $58(48 \%)$ & \\
\hline Male & $41(43 \%)$ & $63(52 \%)$ & \\
\hline First quartile & $23(24 \%)$ & $24(20 \%)$ & \\
\hline Second quartile & $23(24 \%)$ & $36(30 \%)$ & \\
\hline Third quartile & $30(32 \%)$ & 35 (29\%) & \\
\hline Fourth quartile & $19(20 \%)$ & $26(21 \%)$ & \\
\hline Medicaid/Medicare & $21(22 \%)$ & $40(33 \%)$ & \\
\hline Self-pay/other & $10(11 \%)$ & $10(8 \%)$ & \\
\hline Hospital bed size & & & 0.168 \\
\hline Small & $5(5 \%)$ & $14(11 \%)$ & \\
\hline Medium & $16(17 \%)$ & $25(21 \%)$ & \\
\hline Large & $74(78 \%)$ & $82(68 \%)$ & \\
\hline Hospital ownership & & & 0.804 \\
\hline Government, non-federal & $7(7 \%)$ & $12(10 \%)$ & \\
\hline Rural & $4(4 \%)$ & $4(3 \%)$ & \\
\hline
\end{tabular}

$I Q R$, interquartile range; FAP, familial adenomatous polyposis.

Furthermore, the patients in our study are less likely to have comorbid conditions given that our study does not include older patients. Lastly, we excluded any patients undergoing procedures during non-elective admissions and those with a diagnosis of malignancy given that those patients could have potentially required urgent surgery under less than optimal conditions due to obstructive symptoms or bleeding.

We did not include the most recent release of the KID (year 2016) due to the complexities in mixing ICD-9 and ICD-10 data. Our data from 2009 and 2012 predate the widespread implementation of enhanced recovery after surgery (ERAS) protocols over recent years, which have aimed to improve postoperative pain, decrease length of stay, and reduce costs. It is therefore very possible that the increasingly common utilization of standardized perioperative protocols for patients undergoing minimally invasive colorectal surgery may yield demonstrable differences in length of stay between laparoscopic and open approaches. Although we did not appreciate substantial differences in our study, we suspect that future data will demonstrate shorter length of stay, and therefore reduced costs, associated with minimally invasive surgery for 
Table 2 Postoperative complications following prophylactic colectomy for FAP

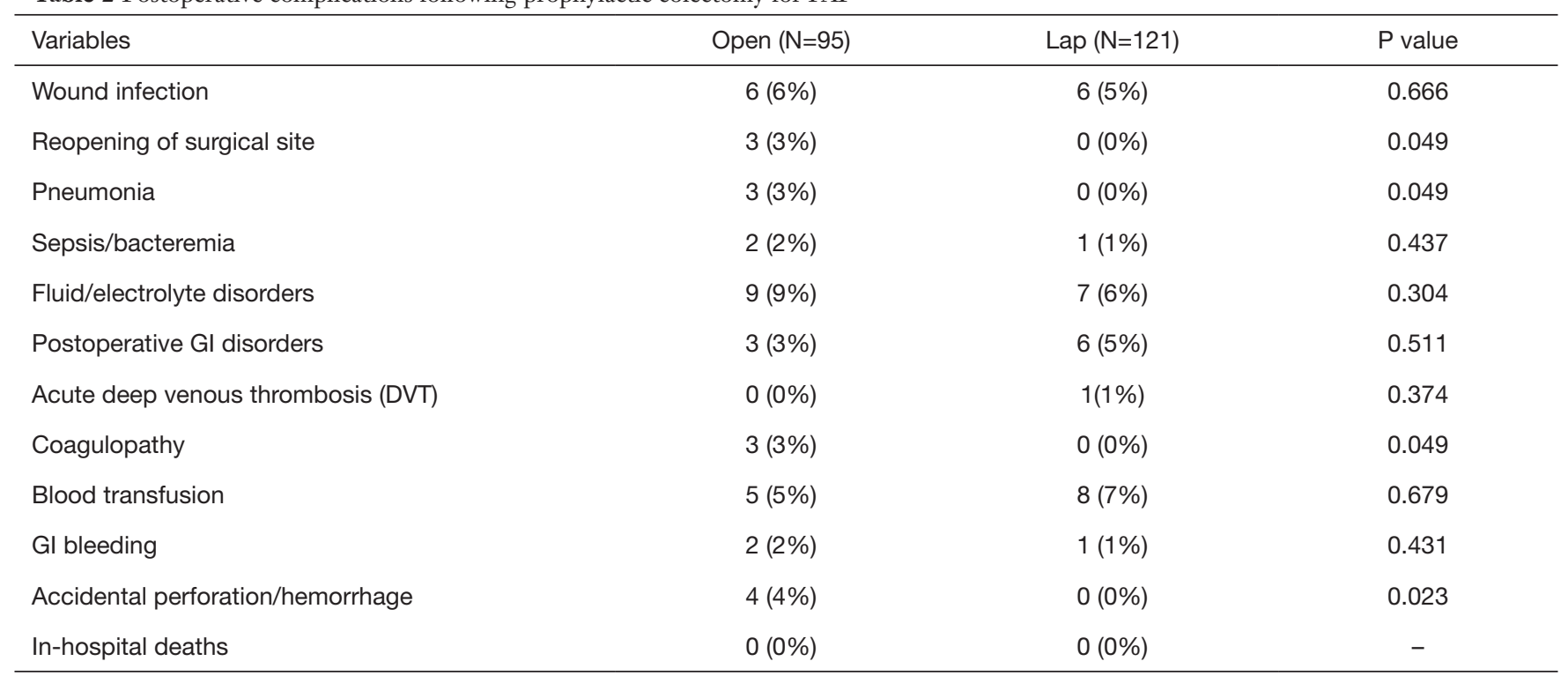

FAP, familial adenomatous polyposis.

Table 3 Associated procedures, length of stay, and cost for prophylactic colectomy

\begin{tabular}{lccc}
\hline Variables & Open $(\mathrm{N}=95)$ & Lap $(\mathrm{N}=121)$ & $\mathrm{P}$ value \\
\hline lleostomy & $70(74 \%)$ & $59(49 \%)$ & $86(71 \%)$ \\
Proctectomy & $86(91 \%)$ & 6 days (IQR: $5-10)$ & $<0.001$ \\
Length of stay (median) & 7 days (IQR: 5-10) & 0.712 \\
Total hospital charges (median) & $\$ 67,334($ IQR: $\$ 42,432-\$ 92,600)$ & $\$ 68,717($ IQR: $\$ 47,912-\$ 115,391)$ & 0.080 \\
\hline
\end{tabular}

$I Q R$, interquartile range.

children with FAP.

An unexpected finding in this study is the predilection for total colectomy over proctocolectomy among the patients that underwent laparoscopic surgery. While controversy remains regarding the necessity and timing of removing the rectum for FAP, it is unclear why the decision to pursue a minimally invasive approach would influence the extent of the upfront surgical resection. One possible explanation is that this group could have simply included more patients with limited polyposis of the rectum for which total abdominal colectomy with subsequent endoscopic management of the rectum is feasible. For such patients, avoidance of proctectomy is recommended when possible to avoid the increased morbidity associated with pelvic surgery in young patients, specifically with regard to fertility and bowel function. On the other hand, it is possible that this difference is secondary to patient selection by the surgeon. Some surgeons may lack the training and technical skills required to safely perform the pelvic dissection laparoscopically and therefore only offer open surgery to those patients requiring total proctocolectomy while reserving laparoscopic surgery for those requiring resection of the colon and not the rectum. Although it is difficult to determine the true cause for this variance in rectal resection rates in our study, we attribute the higher rate of ileostomy creation in the open surgery cohort to the similarly elevated rate of simultaneous proctectomy performed among this group given that most surgeons create a temporary diverting ileostomy at the time of performing restorative proctocolectomy.

Several limitations are inherent to our study due to the nature of utilizing a retrospectively collected administrative database such as KID. Among them are possible errors in data sampling, collection measures, usage of the ICD-9 
coding scheme, and administrative errors during data entry. Additionally, although this is the largest study if its kind for this rare pediatric condition, our analysis captures data for only 216 patients. Therefore, it is possible that type II error may be present in which we did not detect differences that may actually exist. Another major limitation is the lack of data regarding long-term outcomes. Prophylactic colectomy for FAP, whether done in an open or laparoscopic fashion, is performed with the ultimate goal of improving long-term survival by preventing colorectal malignancy. However, since KID is collected from single hospital admissions without follow-up information, our study is unable to compare the oncologic outcomes for patients undergoing these prophylactic procedures. Additionally, we are unable to measure other relevant short-term outcomes beyond hospital discharge such as unplanned hospital readmissions. While the utilization of any administrative inpatient dataset is clearly limited by a lack of granular detail and measures of long-term outcomes, this study provides the largest multi-institutional analysis to date comparing open and laparoscopic techniques for the prophylactic surgical treatment of pediatric patients with FAP.

\section{Conclusions}

Our findings suggest that a laparoscopic approach for prophylactic colon resections can be safely performed in children with FAP and that hospital resource utilization is equivalent compared to conventional open surgery. However, total abdominal colectomy is more commonly performed instead of total proctocolectomy when a laparoscopic approach is utilized. The long-term impact of forgoing resection of the rectum remains to be determined for children with FAP.

\section{Acknowledgments}

Funding: None.

\section{Footnote}

Provenance and Peer Review: This article was commissioned by the editorial office, Translational Gastroenterology and Hepatology for the series "Current Topics in Pediatric General Surgery". The article has undergone external peer review.

Reporting Checklist: The authors have completed the
STROBE reporting checklist. Available at http://dx.doi. org/10.21037/tgh-20-190

Conflicts of Interest: All authors have completed the ICMJE uniform disclosure form (available at http://dx.doi. org/10.21037/tgh-20-190). The series "Current Topics in Pediatric General Surgery" was commissioned by the editorial office without any funding or sponsorship. Dr. SP served as the unpaid Guest Editor of the series. Dr. EAP served as the unpaid Guest Editor of the series and serves as an unpaid editorial board member of Translational Gastroenterology and Hepatology from Jan 2019 to Dec 2020. The authors have no other conflicts of interest to declare.

Ethical Statement: The authors are accountable for all aspects of the work in ensuring that questions related to the accuracy or integrity of any part of the work are appropriately investigated and resolved. The study was conducted in accordance with the Declaration of Helsinki (as revised in 2013). The Institutional Review Board of the University of Miami waived the requirement for approval of this study since the KID contains de-identified, publicly available data and is not considered human subjects research.

Open Access Statement: This is an Open Access article distributed in accordance with the Creative Commons Attribution-NonCommercial-NoDerivs 4.0 International License (CC BY-NC-ND 4.0), which permits the noncommercial replication and distribution of the article with the strict proviso that no changes or edits are made and the original work is properly cited (including links to both the formal publication through the relevant DOI and the license). See: https://creativecommons.org/licenses/by-nc-nd/4.0/.

\section{References}

1. Kennedy RD, Potter DD, Moir CR, et al. The natural history of familial adenomatous polyposis syndrome: a 24 year review of a single center experience in screening, diagnosis, and outcomes. J Pediatr Surg 2014;49:82-6.

2. Vitellaro M, Bonfanti G, Sala P, et al. Laparoscopic colectomy and restorative proctocolectomy for familial adenomatous polyposis. Surg Endosc 2011;25:1866-75.

3. Vitellaro M, Ferrari A, Trencheva K, et al. Is laparoscopic surgery an option to support prophylactic colectomy in adolescent patients with Familial Adenomatous Polyposis (FAP)? Pediatr Blood Cancer 2012;59:1223-8. 
4. Campos FG, Real Martinez CA, Monteiro de Camargo MG, et al. Laparoscopic Versus Open Restorative Proctocolectomy for Familial Adenomatous Polyposis. J Laparoendosc Adv Surg Tech A 2018;28:47-52.

5. Konishi T, Ishida H, Ueno H, et al. Feasibility of laparoscopic total proctocolectomy with ileal pouchanal anastomosis and total colectomy with ileorectal anastomosis for familial adenomatous polyposis: results of a nationwide multicenter study. Int J Clin Oncol 2016;21:953-61.

6. McNicol FJ, Kennedy RH, Phillips RK, et al. Laparoscopic total colectomy and ileorectal anastomosis (IRA), supported by an enhanced recovery programme in cases of familial adenomatous polyposis. Colorectal Dis 2012;14:458-62.

7. Fleming FJ, Francone TD, Kim MJ, et al. A laparoscopic approach does reduce short-term complications in patients undergoing ileal pouch-anal anastomosis. Dis Colon Rectum 2011;54:176-82.

8. Ahmed Ali U, Keus F, Heikens JT, et al. Open versus laparoscopic (assisted) ileo pouch anal anastomosis for ulcerative colitis and familial adenomatous polyposis. Cochrane Database Syst Rev 2009;(1):CD006267.

9. Sinha A, Burns EM, Latchford A, et al. Risk of desmoid formation after laparoscopic versus open colectomy and ileorectal anastomosis for familial adenomatous polyposis. BJS Open 2018;2:452-5.

10. Kauffman JD, Snyder CW, Danielson PD, et al. 30Day Outcomes of Laparoscopic Versus Open Total

doi: 10.21037/tgh-20-190

Cite this article as: Ferrantella A, Saberi RA, Willobee BA, Quiroz HJ, Langshaw AH, Pandya S, Thorson CM, Sola JE, Perez EA. Prophylactic colectomy for children with familial adenomatous polyposis: resource utilization and outcomes for open and laparoscopic surgery. Transl Gastroenterol Hepatol 2021;6:40.
Proctocolectomy with Ileoanal Anastomosis in Children and Young Adults: A Combined Analysis of the National Surgical Quality Improvement Project Pediatric and Adult Databases. J Laparoendosc Adv Surg Tech A 2019;29:402-8.

11. McKenna NP, Potter DD, Bews KA, et al. Ileal-pouch anal anastomosis in pediatric NSQIP: Does a laparoscopic approach reduce complications and length of stay? J Pediatr Surg 2019;54:112-7.

12. Huang CC, Rescorla FJ, Landman MP. Clinical Outcomes After Ileal Pouch-Anal Anastomosis in Pediatric Patients. J Surg Res 2019;234:72-6.

13. White I, Jenkins JT, Coomber R, et al. Outcomes of laparoscopic and open restorative proctocolectomy. Br J Surg 2014;101:1160-5.

14. Zhang H, Hu S, Zhang G, et al. Laparoscopic versus open proctocolectomy with ileal pouch-anal anastomosis. Minim Invasive Ther Allied Technol 2007;16:187-91.

15. KID Database Documentation. Healthcare Cost and Utilization Project (HCUP). September 2019. Agency for Healthcare Research and Quality, Rockville, MD. Available online: www.hcup-us.ahrq.gov/db/nation/kid/ kiddbdocumentation.jsp

16. Kiran RP, da Luz Moreira A, Remzi FH, et al. Factors associated with septic complications after restorative proctocolectomy. Ann Surg 2010;251:436-40.

17. Heuschen UA, Hinz U, Allemeyer EH, et al. Risk factors for ileoanal J pouch-related septic complications in ulcerative colitis and familial adenomatous polyposis. Ann Surg 2002;235:207-16. 